\title{
Total Synthesis of (+)-Peloruside A
}

Meizhong Jin and Richard E. Taylor*

Department of Chemistry and Biochemistry and the Walther Cancer Research Center, University of Notre Dame, Notre Dame, IN 46556

taylor.61@nd.edu

\section{Experimental}

Compound S1:

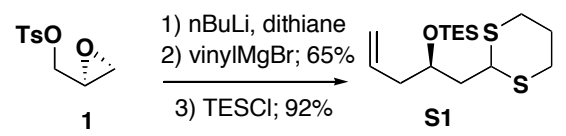

1,3-Dithiane $(2.8 \mathrm{~g}, 23.3 \mathrm{mmol})$ was dissolved in THF $(31 \mathrm{ml})$ and cooled to $-10^{\circ} \mathrm{C} . \mathrm{n}$ Butyllithium (1.4M in hexane, $17 \mathrm{ml}, 23.8 \mathrm{mmol})$ was added and the solution was stirred at $-10^{\circ} \mathrm{C}$ for $2 \mathrm{~h}$ and then cooled to $-78^{\circ} \mathrm{C}$. A solution of (S)-glycidyl tosylate $(5 \mathrm{~g}, 22.0 \mathrm{mmol})$ in $\mathrm{THF}$ $(8 \mathrm{ml})$ was added by cannula, the solution was maintained at $-78^{\circ} \mathrm{C}$ for 4 hours and allowed to warm to room temperature over 2 hours. Saturated aqueous $\mathrm{NaHCO}_{3}$ was added and the mixture was extracted with ether, dried $\left(\mathrm{Na}_{2} \mathrm{SO}_{4}\right)$ and evaporated to give a residue, which was subjected to flash column chromatography to afford the epoxide as a colorless oil $(2.6 \mathrm{~g}, 14.9 \mathrm{mmol})$

To a stirred suspension of $\mathrm{CuI}(500 \mathrm{mg}, 2.6 \mathrm{mmol})$ in THF $(70 \mathrm{ml})$ was added vinylmagnesium bromide $(1.0 \mathrm{M}$ in THF, $22 \mathrm{ml}, 22 \mathrm{mmol})$ at $-50^{\circ} \mathrm{C}$ and the mixture was stirred for $30 \mathrm{~min}$. Then a solution of the epoxide $(2.6 \mathrm{~g}, 14.9 \mathrm{mmol})$ in THF $(10 \mathrm{ml})$ was added by cannula. The resulting mixture was stirred at $-40^{\circ} \mathrm{C}$ for $40 \mathrm{~min}$, allowed to warm to $-10^{\circ} \mathrm{C}$ over $30 \mathrm{~min}$. The reaction mixture was quenched by the addition of saturated aqueous $\mathrm{NH}_{4} \mathrm{Cl}$ and the bulk of THF was removed. The residue was extracted with ether, dried $\left(\mathrm{Na}_{2} \mathrm{SO}_{4}\right)$ and evaporated to give a residue, which was subjected to flash column chromatography to provide secondary alcohol as a colorless oil $(2.8 \mathrm{~g}, 13.7 \mathrm{mmol}, 63 \%)$ : NMR data are identical with literature (F. Yokokawa et al. Tetrahedron 2001, 57, 6311).

To a stirred solution of the alcohol prepared above $(1.4 \mathrm{~g}, 6.8 \mathrm{mmol})$, imidazole $(1.83 \mathrm{mg}$, $27 \mathrm{mmol})$, DMAP $(200 \mathrm{mg}, 1.6 \mathrm{mmol})$ in $5 \mathrm{ml}$ DMF was added TESCl $(1.6 \mathrm{ml}, 9.5 \mathrm{mmol})$ at $0{ }^{\circ} \mathrm{C}$. The mixture was then stirred at r. t. for $12 \mathrm{~h}$, after TLC show the completion of reaction, $20 \mathrm{ml}$ of aq. $\mathrm{NaHCO}_{3}$ was added and the mixture was extracted with ether, dried $\left(\mathrm{Na}_{2} \mathrm{SO}_{4}\right)$ and evaporated to give a residue, which was subjected to flash column chromatography to provide (-)-S1 $(2.1 \mathrm{~g}$, 98\%) as a colorless oil. $[\alpha]_{\mathrm{D}}^{22}=-28.8^{\circ}\left(\mathrm{c} 0.13, \mathrm{CHCl}_{3}\right)$; IR (thin film, $\left.\mathrm{cm}^{-1}\right) 742,915,1005,1088$, $1240,1275,1373,1422,1458,1640,2876,2904,2953 ;{ }^{1} \mathrm{H}$ NMR $\left(300 \mathrm{MHz}, \mathrm{CDCl}_{3}\right) \delta_{\mathrm{H}} 0.63(6 \mathrm{H}$, q, $J=8.1 \mathrm{~Hz}), 0.97(9 \mathrm{H}, \mathrm{t}, J=8.1 \mathrm{~Hz}), 1.79-1.94(3 \mathrm{H}, \mathrm{m}), 2.05-2.15(1 \mathrm{H}, \mathrm{m}), 2.22-2.27(2 \mathrm{H}, \mathrm{m})$, 2.78-2.93 (4H, m), 3.98-4.06 (1H, m), $4.11(1 \mathrm{H}, \mathrm{dd}, J=6.0 \mathrm{~Hz}, 8.4 \mathrm{~Hz}), 5.03-5.09(2 \mathrm{H}, \mathrm{m}), 5.71-$ $5.85(1 \mathrm{H}, \mathrm{m}){ }^{13} \mathrm{C} \mathrm{NMR}\left(75 \mathrm{MHz}, \mathrm{CDCl}_{3}\right) ; \delta_{\mathrm{C}} 5.48,7.09,26.35,30.23,30.71,42.57,42.84,44.21$, 68.64, 117.578, 134.545; HRMS calc. for $\mathrm{C}_{15} \mathrm{H}_{30} \mathrm{OSSi}[\mathrm{M}+\mathrm{H}]$ 319.1586, found 319.1587.

Compound 4:

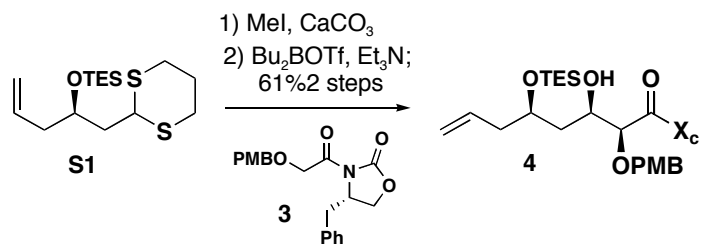

A stirred mixture of $\mathbf{S} \mathbf{1}(116 \mathrm{mg}, 0.36 \mathrm{mmol}), \mathrm{MeI}(0.23 \mathrm{ml}, 3.7 \mathrm{mmol})$ and $\mathrm{CaCO}_{3}(190 \mathrm{mg}$, 
$1.9 \mathrm{mmol})$ in $5 \mathrm{ml} \mathrm{MeCN} / \mathrm{H}_{2} \mathrm{O}(\mathrm{v}: \mathrm{v}=9: 1)$ was heated at $45^{\circ} \mathrm{C}$ for $2.5 \mathrm{~h}$, cool down to room temperature, the bulk of $\mathrm{MeCN}$ was removed. Then the mixture was extracted with ether, dry $\left(\mathrm{Na}_{2} \mathrm{SO}_{4}\right)$ and evaporated to give a residue, which was subjected to flash column chromatography to provide aldehyde 2 and was directly used for the next step.

To a solution of oxazolidinone $3(160 \mathrm{mg}, 0.45 \mathrm{mmol})$ in $3 \mathrm{ml}$ of dry toluene was added $\mathrm{Et}_{3} \mathrm{~N}$ $(0.07 \mathrm{ml}, 0.49 \mathrm{mmol})$ followed by $\mathrm{Bu}_{2} \mathrm{BOTf}\left(1 \mathrm{M}\right.$ in $\left.\mathrm{CH}_{2} \mathrm{Cl}_{2}, 0.45 \mathrm{ml}, 0.45 \mathrm{mmol}\right)$ at $-50^{\circ} \mathrm{C}$. After stirring at $-50^{\circ} \mathrm{C}$ for $1.5 \mathrm{~h}$, aldehyde 2 in $1 \mathrm{ml}$ of toluene was added via cannula. The resulting mixture was warmed to $-30^{\circ} \mathrm{C}$ over $30 \mathrm{~min}$ and held at $-30^{\circ} \mathrm{C}$ for $2 \mathrm{~h}$. The reaction was quenched by adding $0.8 \mathrm{ml}$ of $\mathrm{pH} 7$ phosphate buffer followed by $0.8 \mathrm{ml}$ of methanol and $0.8 \mathrm{ml}$ of etrahydrofuran. After $5 \mathrm{~min}, 0.4 \mathrm{~mL}$ of $30 \%$ aqueous hydrogen peroxide was added (dropwise). The mixture was then warmed up to $0^{\circ} \mathrm{C}$ and stirred at $0^{\circ} \mathrm{C}$ for $1 \mathrm{~h}$, concentrated by rotary evaporation and diluted with aqueous $\mathrm{NaHCO}_{3}$. This mixture was extracted with ethyl acetate, dried $\left(\mathrm{Na}_{2} \mathrm{SO}_{4}\right)$ and evaporated to give a residue, which was subjected to flash column chromatography to provide (+)-4 $(125 \mathrm{mg}, 61 \%, 2$ steps $)$ as a colorless oil. $[\alpha]^{22}{ }_{\mathrm{D}}=+7.05^{\circ}(\mathrm{c} 0.02$, $\mathrm{CHCl}_{3}$ ); IR (thin film, $\mathrm{cm}^{-1}$ ) 745, 823, 1108, 1248, 1390, 1456, 1514, 1612, 1708, 1781, 2954, 3502; ${ }^{1} \mathrm{H}$ NMR $\left(300 \mathrm{MHz}, \mathrm{CDCl}_{3}\right) \delta_{\mathrm{H}} 0.64(6 \mathrm{H}, \mathrm{q}, J=7.8 \mathrm{~Hz}), 0.97(9 \mathrm{H}, \mathrm{t}, J=7.8 \mathrm{~Hz}), 1.71(1 \mathrm{H}$, ddd, $J=2.4,6.0,14.4 \mathrm{~Hz}), 1.87(1 \mathrm{H}$, ddd, $J=6.9,9.9,14.4 \mathrm{~Hz}), 2.22-2.38(2 \mathrm{H}, \mathrm{m}), 2.71(1 \mathrm{H}$, dd, $J=9.9,13.5 \mathrm{~Hz}), 2.96(1 \mathrm{H}, \mathrm{d}, J=5.7 \mathrm{~Hz}), 3.27(1 \mathrm{H}, \mathrm{dd}, J=3.3,13.5 \mathrm{~Hz}), 3.81(3 \mathrm{H}, \mathrm{s}), 3.96-4.12$ $(2 \mathrm{H}, \mathrm{m}), 4.15-4.27(2 \mathrm{H}, \mathrm{m}), 4.51(1 \mathrm{H}, \mathrm{d}, J=11.4 \mathrm{~Hz}), 4.67-4.74(2 \mathrm{H}, \mathrm{m}), 5.07-5.15(2 \mathrm{H}, \mathrm{m})$, 5.75-5.89 $(1 \mathrm{H}, \mathrm{m}), 6.90(2 \mathrm{H}, \mathrm{d}, J=8.7 \mathrm{~Hz}), 7.22-7.40(7 \mathrm{H}, \mathrm{m}),{ }^{13} \mathrm{C} \mathrm{NMR}\left(75 \mathrm{MHz}, \mathrm{CDCl}_{3}\right) ; \delta_{\mathrm{C}}$ 5.18, 7.04, 37.89, 39.93, 42.01, 55.44, 55.85, 67.00, 71.15, 71.21, 72.99, 79.45, 114.02, 117.69, $127.59,129.18,129.42,129.59,130.38,134.52,135.38,153.55,159.79,170.78$; HRMS calc. for $\mathrm{C}_{32} \mathrm{H}_{45} \mathrm{NO}_{7} \mathrm{Si}[\mathrm{M}+\mathrm{H}]$ 584.3044, found 584.3027.

Compound 5:

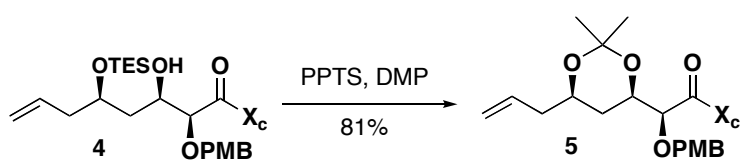

To a stirred solution of $4(15 \mathrm{mg}, 0.024 \mathrm{mmol})$ and 2,2-dimethoxylpropane (7.4mg, $0.071 \mathrm{mmol})$ in $1.5 \mathrm{ml} \mathrm{CH} \mathrm{Cl}_{2}$ was added PPTS $(0.6 \mathrm{mg}, 0.0024 \mathrm{mmol})$ at $0^{\circ} \mathrm{C}$. The stirred mixture was then warm up to r. t. slowly and stirred at $\mathrm{r}$. t. for $2 \mathrm{~h}$. The reaction mixture was concentrated by rotary evaporation to give a residue, which was purified by column chromatography to afford $(+)-5(10 \mathrm{mg}, 81 \%)$ as a colorless oil, $[\alpha]^{22}{ }_{\mathrm{D}}=+39.1^{\circ}\left(\mathrm{c} 0.002, \mathrm{CHCl}_{3}\right)$; IR (thin film, $\left.\mathrm{cm}^{-1}\right) 701$, $1032,1110,1248,1380,1513,1612,1705,1781,2928 ;{ }^{1} \mathrm{H}$ NMR $\left(300 \mathrm{MHz}, \mathrm{CDCl}_{3}\right) \delta_{\mathrm{H}} 1.28-1.49$ $(1 \mathrm{H}, \mathrm{m}), 1.34(3 \mathrm{H}, \mathrm{s}), 1.42(3 \mathrm{H}, \mathrm{s}), 1.57-1.63(1 \mathrm{H}, \mathrm{m}), 2.12-2.22(1 \mathrm{H}, \mathrm{m}), 2.27-2.36(1 \mathrm{H}, \mathrm{m}), 2.69$ $(1 \mathrm{H}, \mathrm{dd}, \mathrm{J}=13.5,9.6 \mathrm{~Hz}), 3.22(1 \mathrm{H}, \mathrm{dd}, \mathrm{J}=13.5,3.3 \mathrm{~Hz}), 3.81(3 \mathrm{H}, \mathrm{s}), 3.82-3.92(1 \mathrm{H}, \mathrm{m}), 4.14-$ $4.19(2 \mathrm{H}, \mathrm{m}), 4.30-4.37(1 \mathrm{H}, \mathrm{m}), 4.56-4.69(3 \mathrm{H}, \mathrm{m}), 5.06-5.14(2 \mathrm{H}, \mathrm{m}), 5.48(1 \mathrm{H}, \mathrm{d}, \mathrm{J}=5.4 \mathrm{~Hz})$, 5.73-5.87 (1H, m), 6.89-7.37 (9H, m); ${ }^{13} \mathrm{C}$ NMR $\left(75 \mathrm{MHz}, \mathrm{CDCl}_{3}\right) \delta_{\mathrm{C}} 19.8,30.1,30.9,38.3,40.9$, $55.5,56.1,66.8,68.8,69.9,73.3,77.0,98.9,113.9,117.4,127.6,129.2,129.6,130.5,134.2$, 135.5, 153.5, 159.7, 170.6; HRMS calc. for $\mathrm{C}_{29} \mathrm{H}_{35} \mathrm{NO}_{7}[\mathrm{M}-\mathrm{H}]^{+}$508.2335, found 508.2338. 
Compound 6:

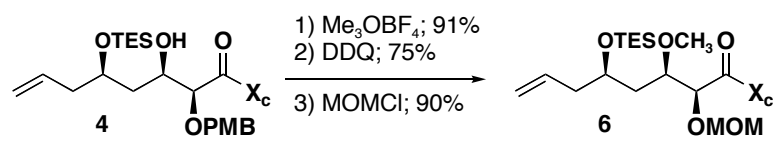

To a stirred solution of 4 (120mg, $0.2 \mathrm{mmol})$ in $5 \mathrm{ml} \mathrm{CH}_{2} \mathrm{Cl}_{2}$ was added proton sponge $(642 \mathrm{mg}, 3.0 \mathrm{mmol})$ followed by trimethyloxonium tetrafluoroborate $(444 \mathrm{mg}, 3.0 \mathrm{mmol})$ at $0^{\circ} \mathrm{C}$. After stirring $15 \mathrm{~h}$ at room temperature, the reaction was quenched with aq. $\mathrm{NaHCO}_{3}$ and extracted with $\mathrm{CH}_{2} \mathrm{Cl}_{2}$. The extract was washed with sat. aq. $\mathrm{NaCl}$, dried $\left(\mathrm{Na}_{2} \mathrm{SO} 4\right)$ and evaporated to give a residue, which was filtered through a short column to provide a crude material which was directly used for the next step.

To a stirred solution of crude material prepared above in $2 \mathrm{ml} \mathrm{CH}_{2} \mathrm{Cl}_{2}$ was added $\mathrm{H}_{2} \mathrm{O}$ $(0.1 \mathrm{ml})$ followed by DDQ $(143 \mathrm{mg}, 0.63 \mathrm{mmol})$ at $0{ }^{\circ} \mathrm{C}$, after stirring at $0{ }^{\circ} \mathrm{C}$ for $20 \mathrm{~min}$, the ice bath was removed and the mixture was stirred at r. t. for $2 \mathrm{~h}$. The mixture was then diluted with 15 $\mathrm{ml}$ of saturated aqueous sodium bicarbonate and extracted with $\mathrm{CH}_{2} \mathrm{Cl}_{2}$. The organic layers were combined, dried $\left(\mathrm{Na}_{2} \mathrm{SO}_{4}\right)$, and evaporated to give a residue which was then dissolved in $1 \mathrm{ml}$ $\mathrm{CH}_{2} \mathrm{Cl}_{2}$, to this solution was added DIPEA $(348 \mathrm{mg}, 2.7 \mathrm{mmol})$ followed by $\mathrm{MOMCl}(145 \mathrm{mg}$, $1.8 \mathrm{mmol})$ at $0^{\circ} \mathrm{C}$. The resulting mixture was warmed up to r. t. slowly and stirred at $\mathrm{r}$. t. for $15 \mathrm{~h}$. Then diluted with $5 \mathrm{ml}$ of saturated aqueous sodium bicarbonate and extracted with ether. The organic layers were combined, dried $\left(\mathrm{Na}_{2} \mathrm{SO}_{4}\right)$ and evaporated to give a residue, which was purified by column chromatography afforded $(+)-6(62 \mathrm{mg}, 62 \%, 3$ steps $)$ as a colorless oil, $[\alpha]^{22}=44.86^{\circ}\left(\mathrm{c} 0.007, \mathrm{CHCl}_{3}\right)$; IR (thin film, $\left.\mathrm{cm}^{-1}\right) 702,745,917,1007,1048,1107,1153,1211$, $1349,1387,1455,1709,1783,2877,2954 ;{ }^{1} \mathrm{H}$ NMR $\left(300 \mathrm{MHz}, \mathrm{CDCl}_{3}\right) \delta_{\mathrm{H}} 0.60(6 \mathrm{H}, \mathrm{q}, J=8.1$ $\mathrm{Hz}), 0.95(9 \mathrm{H}, \mathrm{t}, J=8.1 \mathrm{~Hz}), 1.78(2 \mathrm{H}, \mathrm{t}, J=6.3 \mathrm{~Hz}), 2.17-2.37(2 \mathrm{H}, \mathrm{m}), 2.78(1 \mathrm{H}, \mathrm{dd}, J=9.6$, $13.5 \mathrm{~Hz}), 3.34(1 \mathrm{H}, \mathrm{dd}, J=3.3,9.6 \mathrm{~Hz}), 3.39(3 \mathrm{H}, \mathrm{s}), 3.40(3 \mathrm{H}, \mathrm{s}), 3.70-3.76(1 \mathrm{H}, \mathrm{m}), 3.86-3.94$ $(1 \mathrm{H}, \mathrm{m}), 4.15-4.22(2 \mathrm{H}, \mathrm{m}), 4.60-4.67(1 \mathrm{H}, \mathrm{m}), 4.69(1 \mathrm{H}, \mathrm{d}, J=6.9 \mathrm{~Hz}), 4.79(1 \mathrm{H}, \mathrm{d}, J=6.9 \mathrm{~Hz})$, 5.02-5.11 (2H, m), $5.48(1 \mathrm{H}, \mathrm{d}, J=4.8 \mathrm{~Hz}), 5.77-5.92(1 \mathrm{H}, \mathrm{m}), 7.23-7.37(5 \mathrm{H}, \mathrm{m}) ;{ }^{13} \mathrm{C}$ NMR $(75$ $\left.\mathrm{MHz}, \mathrm{CDCl}_{3}\right) \delta_{\mathrm{C}} 5.21,7.19,37.72,37.79,41.65,55.98,56.58,58.78,66.63,69.34,77.20,78.60$, 97.77, 117.44, 127.64, 129.27, 129.74, 135.26, 135.51, 153.43, 171.23 ; HRMS calc. for $\mathrm{C}_{27} \mathrm{H}_{43} \mathrm{NO}_{7} \mathrm{Si}[\mathrm{M}+\mathrm{H}]^{+}$522.2887, found 522.2862.

Compound 7:

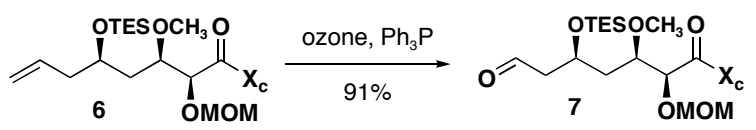

To a stirred solution of $6(125 \mathrm{mg}, 0.24 \mathrm{mmol})$ in $8 \mathrm{ml} \mathrm{CH}_{2} \mathrm{Cl}_{2}$ was added pyridine (50mg, $0.63 \mathrm{mmol})$ at $-78^{\circ} \mathrm{C}$, ozone was bubbled through the solution until TLC shows the completion of reaction. $\mathrm{PPh}_{3}(125 \mathrm{mg}, 0.48 \mathrm{mmol})$ was added and the mixture was warmed up to room temperature slowly and stirred at room temperature for 4 hours. Then the reaction mixture was diluted with aq. $\mathrm{NaHCO}_{3}$ and extracted with $\mathrm{CH}_{2} \mathrm{Cl}_{2}$, dried $\left(\mathrm{Na}_{2} \mathrm{SO}_{4}\right)$ and evaporated to give a residue, which was subjected to a short column to provide aldehyde 7 as colorless oil and was directly used for the next step. ${ }^{1} \mathrm{H}$ NMR $\left(300 \mathrm{MHz}, \mathrm{CDCl}_{3}\right) \delta_{\mathrm{H}} 0.62(6 \mathrm{H}, \mathrm{q}, J=8.1 \mathrm{~Hz}), 0.97(9 \mathrm{H}$, t, $J=8.1 \mathrm{~Hz}), 1.89(2 \mathrm{H}, \mathrm{t}, J=6.6 \mathrm{~Hz}), 2.55-2.70(2 \mathrm{H}, \mathrm{m}), 2.78(1 \mathrm{H}, \mathrm{dd}, J=9.9,13.5 \mathrm{~Hz}), 3.36$ $(1 \mathrm{H}, \mathrm{dd}, J=3.0,13.5 \mathrm{~Hz}), 3.42(3 \mathrm{H}, \mathrm{s}), 3.43(3 \mathrm{H}, \mathrm{s}), 3.71-3.79(1 \mathrm{H}, \mathrm{m}), 4.19-4.24(2 \mathrm{H}, \mathrm{m}), 4.40-$ $4.48(1 \mathrm{H}, \mathrm{m}), 4.62-4.69(1 \mathrm{H}, \mathrm{m}), 4.71(1 \mathrm{H}, \mathrm{d}, J=6.9 \mathrm{~Hz}), 4.81(1 \mathrm{H}, \mathrm{d}, J=6.9 \mathrm{~Hz}), 5.55(1 \mathrm{H}, \mathrm{d}, J$ $=4.5 \mathrm{~Hz}), 7.23-7.40(5 \mathrm{H}, \mathrm{m}), 9.81(1 \mathrm{H}, \mathrm{t}, J=2.4 \mathrm{~Hz}) ;{ }^{13} \mathrm{C} \mathrm{NMR}\left(75 \mathrm{MHz}, \mathrm{CDCl}_{3}\right) \delta_{\mathrm{C}} 5.08,7.07$, $37.69,38.55,50.89,56.00,56.58,58.98,65.52,66.68,76.31,78.37,97.73,127.67,129.28$, 
$129.73,135.44,153.75,171.14,202.26$.

Compound 9:

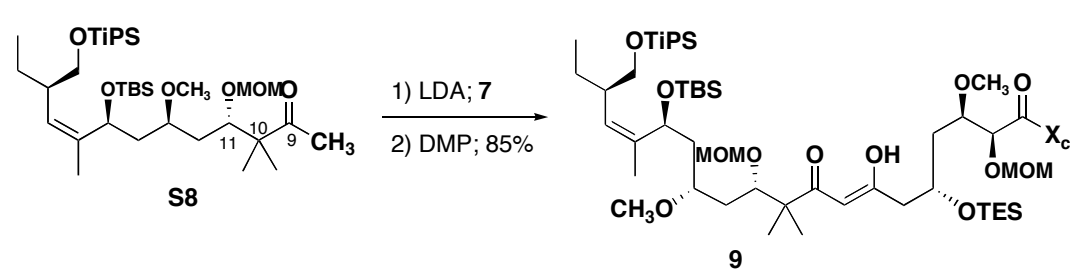

To a stirred solution of diisopropylamime $(41 \mathrm{mg}, 0.40 \mathrm{mmol})$ in $5 \mathrm{ml}$ THF was added BuLi $(1.7 \mathrm{M}$ in hexane, $0.23 \mathrm{ml}, 0.39 \mathrm{mmol})$ at $0^{\circ} \mathrm{C}$, the mixture was stirred at $0^{\circ} \mathrm{C}$ for $30 \mathrm{~min}$. Cooled to $-78^{\circ} \mathrm{C}$, ketone $\mathbf{S 8}(230 \mathrm{mg}, 0.36 \mathrm{mmol})$ in $2 \mathrm{ml}$ THF was added, the reaction was stirred at $-78^{\circ} \mathrm{C}$ for 1 hour and aldehyde $7 \mathrm{in} 3 \mathrm{ml}$ THF was added. The reaction was stirred at $-78^{\circ} \mathrm{C}$ for $1 \mathrm{~min}$ and quenched by $1 \mathrm{ml}$ aqueous $\mathrm{NH}_{4} \mathrm{Cl}$. The resulting mixture was warmed up to room temperature in 1 hour and extracted with $\mathrm{CH}_{2} \mathrm{Cl}_{2}$, dried $\left(\mathrm{Na}_{2} \mathrm{SO}_{4}\right)$ and evaporated to give a residue, which was subjected to a short column to provide a mixture of two diastereomers $(165 \mathrm{mg})$ as colorless oil. This material was then dissolved in $5 \mathrm{ml}$ of $\mathrm{CH}_{2} \mathrm{Cl}_{2}$ and was cannulated to a stirred mixture of Dess-Martin periodinane (360mg, $0.85 \mathrm{mmol})$ and pyridine $(167 \mathrm{mg}, 2.1 \mathrm{mmol})$ in $10 \mathrm{ml} \mathrm{CH}_{2} \mathrm{Cl}_{2}$ at $0^{\circ} \mathrm{C}$. After stirring at the same temperature for 4 hours, aqueous $\mathrm{Na}_{2} \mathrm{~S}_{2} \mathrm{O}_{3}$ was added followed by aqueous $\mathrm{NaHCO}_{3}$. Extracted with $\mathrm{CH}_{2} \mathrm{Cl}_{2}$, the organic layers were combined, dried $\left(\mathrm{Na}_{2} \mathrm{SO}_{4}\right)$ and evaporated to give a residue, which was purified by column chromatography to afford (+)-9 $(141 \mathrm{mg}, 85 \%)$ as a colorless oil, $[\alpha]^{22}=+25.4^{\circ}\left(\mathrm{c}, 0.01, \mathrm{CHCl}_{3}\right)$; IR (thin film, $\left.\mathrm{cm}^{-1}\right) 688,700$, $745,775,836,882,920,1101,1153,1210,1250,1387,1460,1604,1708,1784,2866,2954 ;{ }^{1} \mathrm{H}$ $\operatorname{NMR}\left(500 \mathrm{MHz}, \mathrm{CDCl}_{3}\right) \delta_{\mathrm{H}} 0.01(3 \mathrm{H}, \mathrm{s}), 0.07(3 \mathrm{H}, \mathrm{s}), 0.62(6 \mathrm{H}, \mathrm{q}, J=8.0 \mathrm{~Hz}), 0.83(3 \mathrm{H}, \mathrm{t}, J=$ $7.0 \mathrm{~Hz}), 0.90(9 \mathrm{H}, \mathrm{s}), 0.94(9 \mathrm{H}, \mathrm{t}, J=8.0 \mathrm{~Hz}), 1.05-1.09(21 \mathrm{H}, \mathrm{m}), 1.10(3 \mathrm{H}, \mathrm{s}), 1.16-1.23(5 \mathrm{H}$, $\mathrm{m}), 1.38-1.45(1 \mathrm{H}, \mathrm{m}), 1.63-1.77(5 \mathrm{H}, \mathrm{m}), 1.85(2 \mathrm{H}, \mathrm{t}, J=6.0 \mathrm{~Hz}), 2.12-2.19(1 \mathrm{H}, \mathrm{m}), 2.34-2.41$ $(1 \mathrm{H}, \mathrm{m}), 2.45(1 \mathrm{H}, \mathrm{dd}, J=8.0,14.0 \mathrm{~Hz}), 2.55(1 \mathrm{H}, \mathrm{dd}, J=5.0,14.0 \mathrm{~Hz}), 2.75-2.83(1 \mathrm{H}, \mathrm{m}), 3.30$ $(3 \mathrm{H}, \mathrm{s}), 3.35(3 \mathrm{H}, \mathrm{s}), 3.34-3.41(1 \mathrm{H}, \mathrm{m}), 3.42(3 \mathrm{H}, \mathrm{s}), 3.45(3 \mathrm{H}, \mathrm{s}), 3.43-3.51(2 \mathrm{H}, \mathrm{m}), 3.68-3.71$ $(1 \mathrm{H}, \mathrm{m}), 3.74-3.79(1 \mathrm{H}, \mathrm{m}), 4.05(1 \mathrm{H}, \mathrm{dd}, J=1.5,10.5 \mathrm{~Hz}), 4.18-4.25(2 \mathrm{H}, \mathrm{m}), 4.31-4.40(1 \mathrm{H}$, $\mathrm{m}), 4.51(1 \mathrm{H}, \mathrm{dd}, J=2.5,9.5 \mathrm{~Hz}), 4.58-4.69(3 \mathrm{H}, \mathrm{m}), 4.72(1 \mathrm{H}, \mathrm{d}, J=7.0 \mathrm{~Hz}), 4.81(1 \mathrm{H}, \mathrm{d}, J=$ $7.0 \mathrm{~Hz}), 4.96(1 \mathrm{H}, \mathrm{d}, J=9.5 \mathrm{~Hz}), 5.51(1 \mathrm{H}, \mathrm{d}, J=4.5 \mathrm{~Hz}), 5.70(1 \mathrm{H}, \mathrm{s}), 7.26-7.38(5 \mathrm{H}, \mathrm{m}) ;{ }^{13} \mathrm{C}$ NMR $\left(125 \mathrm{MHz}, \mathrm{CDCl}_{3}\right) \delta_{\mathrm{C}}-4.75,-4.72,5.02,7.11,12.20,15.53,18.27,18.31,20.98,22.25$, $25.04,25.11,29.95,37.11,37.71,38.92,40.68,46.69,54.87,55.97,56.33,56.55,59.09,66.12$, $66.24,66.65,67.21,67.66,74.17,74.40,76.73,78.51,80.43,97.73,98.85,98.97,127.04,127.64$, $129.26,129.74,135.47,139.19,153.46,171.14,192.52,199.43$.

Compound 10:
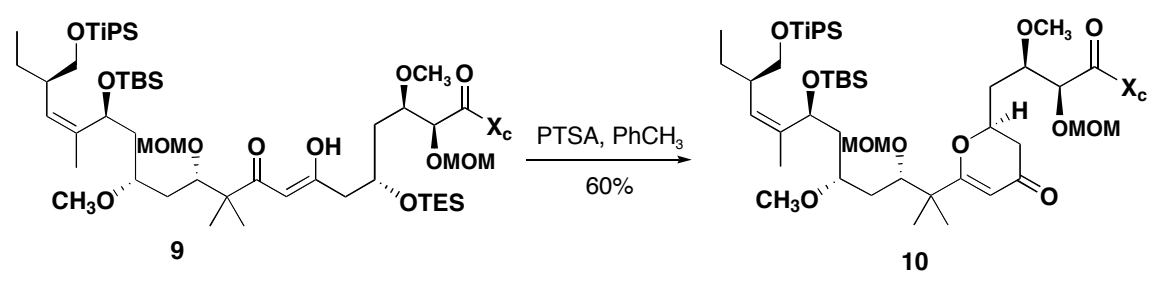

To a stirred solution of $9(152 \mathrm{mg}, 0.13 \mathrm{mmol})$ in $10 \mathrm{ml}$ toluene was added p-toluenesulfonic acid $(1.9 \mathrm{mg}, 0.01 \mathrm{mmol})$ and the mixture was heated at $45^{\circ} \mathrm{C}$ for 2.5 hours. After TLC shows the completion of reaction, aqueous $\mathrm{NaHCO}_{3}$ was added. Extracted with ethyl acetate, the organic layers were combined, dried $\left(\mathrm{Na}_{2} \mathrm{SO}_{4}\right)$ and evaporated to give a residue, which was purified by column chromatography to afford (-)-10 $(81 \mathrm{mg}, 62 \%)$ as a colorless oil, $[\alpha]^{22}{ }^{2}=-36.69^{\circ}(\mathrm{c}, 0.003$, 
$\mathrm{CHCl}_{3}$ ); IR (thin film, $\mathrm{cm}^{-1}$ ) 688, 701, 775, 836, 882, 920, 1035, 1103, 1196, 1211, 1249, 1349, $1388,1463,1596,1666,1707,1782,2865,2893,2944 ;{ }^{1} \mathrm{H}$ NMR $\left(500 \mathrm{MHz}, \mathrm{CDCl}_{3}\right) \delta_{\mathrm{H}} 0.01(3 \mathrm{H}$, s), $0.07(3 \mathrm{H}, \mathrm{s}), 0.82(3 \mathrm{H}, \mathrm{t}, J=7.5 \mathrm{~Hz}), 0.91(9 \mathrm{H}, \mathrm{s}), 1.05-1.09(21 \mathrm{H}, \mathrm{m}), 1.10(3 \mathrm{H}, \mathrm{s}), 1.13-1.23$ $(5 \mathrm{H}, \mathrm{m}), 1.40-1.45(1 \mathrm{H}, \mathrm{m}), 1.66-1.80(5 \mathrm{H}, \mathrm{m}), 2.10-2.25(3 \mathrm{H}, \mathrm{m}), 2.31-2.39(1 \mathrm{H}, \mathrm{m}), 2.42(1 \mathrm{H}$, dd, $J=4.0,16.5 \mathrm{~Hz}), 2.50(1 \mathrm{H}, \mathrm{dd}, J=14.0,16.5 \mathrm{~Hz}), 2.82(1 \mathrm{H}, \mathrm{dd}, J=10.0,13.5 \mathrm{~Hz}), 3.29(3 \mathrm{H}$, s), $3.34(3 \mathrm{H}, \mathrm{s}), 3.38(1 \mathrm{H}, \mathrm{dd}, J=4.0,13.5 \mathrm{~Hz}), 3.43(3 \mathrm{H}, \mathrm{s}), 3.44(3 \mathrm{H}, \mathrm{s}), 3.44-3.51(2 \mathrm{H}, \mathrm{m})$, $3.69(1 \mathrm{H}, \mathrm{dd}, J=4.0,10.0 \mathrm{~Hz}), 3.86-3.89(1 \mathrm{H}, \mathrm{m}), 4.05(1 \mathrm{H}, \mathrm{d}, J=9.5 \mathrm{~Hz}), 4.21(1 \mathrm{H}, \mathrm{dd}, J=2.5$, $9.0 \mathrm{~Hz}), 4.25(1 \mathrm{H}, \mathrm{t}, J=9.0 \mathrm{~Hz}), 4.49(1 \mathrm{H}, \mathrm{d}, J=8.0 \mathrm{~Hz}), 4.58-4.64(2 \mathrm{H}, \mathrm{m}), 4.67(1 \mathrm{H}, \mathrm{d}, J=6.5$ $\mathrm{Hz}), 4.70-4.75(2 \mathrm{H}, \mathrm{m}), 4.83(1 \mathrm{H}, \mathrm{d}, J=6.5 \mathrm{~Hz}), 4.97(1 \mathrm{H}, \mathrm{d}, J=10.5 \mathrm{~Hz}), 5.50(1 \mathrm{H}, \mathrm{s}), 5.65$ $(1 \mathrm{H}, \mathrm{d}, J=4.5 \mathrm{~Hz}), 7.26-7.38(5 \mathrm{H}, \mathrm{m}) ;{ }^{13} \mathrm{C} \mathrm{NMR}\left(125 \mathrm{MHz}, \mathrm{CDCl}_{3}\right) \delta_{\mathrm{C}}-4.75,-4.43,12.19$, $12.31,18.27,18.28,18.32,21.73,21.87,25.07,26.06,35.27,37.03,37.71,40.73,41.20,42.18$, $45.03,55.11,56.01,56.42,56.69,58.85,66.32,66.70,67.64,74.39,75.42,78.05,80.58,97.81$, $99.09,103.34,127.01,127.67,129.28,129.75,135.43,139.25,153.49,170.82,182.90,193.65$; HRMS calc. for $\mathrm{C}_{55} \mathrm{H}_{95} \mathrm{NO}_{13} \mathrm{Si}_{2}[\mathrm{M}+\mathrm{Na}]^{+} 1056.6240$, found 1056.6217 .

Compound S10:

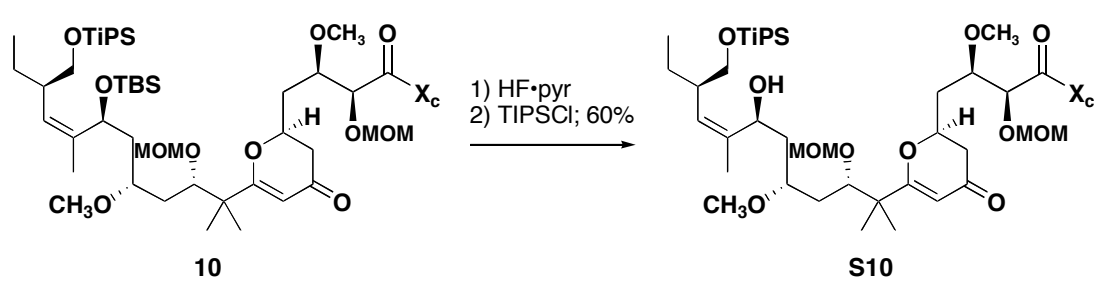

To a stirred solution of $\mathbf{1 0}(60 \mathrm{mg}, 0.058 \mathrm{mmol})$ in $5 \mathrm{ml}$ mixture solvent $\left(\mathrm{MeCN} / \mathrm{H}_{2} \mathrm{O}, \mathrm{v} / \mathrm{v}=\right.$ 5:1) was added $\mathrm{HF} /$ Pyridine complex $(440 \mathrm{mg}, 4.4 \mathrm{mmol})$ at $0^{\circ} \mathrm{C}$ and the mixture was stirred at room temperature for 15 hours. After TLC shows the completion of reaction, aqueous $\mathrm{NaHCO}_{3}$ was added. Extracted with ethyl acetate, the organic layers were combined, dried $\left(\mathrm{Na}_{2} \mathrm{SO}_{4}\right)$ and evaporated to give the crude diol, which was then dissolved in $1 \mathrm{ml} \mathrm{CH}_{2} \mathrm{Cl}_{2}$. To this solution was added imidazole $(30 \mathrm{mg}, 0.44 \mathrm{mmol})$, DMAP $(1.8 \mathrm{mg}, 0.015 \mathrm{mmol})$ followed by TIPSCl $(40 \mathrm{mg}$, $0.20 \mathrm{mmol}$ ) at $0^{\circ} \mathrm{C}$, the resulting mixture was stirred at room temperature for about 10 hours. After TLC shows the completion of reaction, aqueous $\mathrm{NaHCO}_{3}$ was added. Extracted with ethyl acetate, the organic layers were combined, dried $\left(\mathrm{Na}_{2} \mathrm{SO}_{4}\right)$ and evaporated to give a residue, which was purified by column chromatography to provide (-)-S10 (34mg, 65\%, 2 steps) as a colorless oil, $[\alpha]_{\mathrm{D}}^{22}=-34.0^{\circ}$ (c $0.01, \mathrm{CHCl}_{3}$ ); IR (thin film, $\mathrm{cm}^{-1}$ ) 882, 919, 1037, 1100, 1211, $1388,1463,1594,1662,1708,1781,2866,2943,3468 ;{ }^{1} \mathrm{H}$ NMR $\left(500 \mathrm{MHz}, \mathrm{CDCl}_{3}\right) \delta_{\mathrm{H}} 0.85(3 \mathrm{H}$, $\mathrm{t}, J=7.5 \mathrm{~Hz}), 1.04-1.09(21 \mathrm{H}, \mathrm{m}), 1.10(3 \mathrm{H}, \mathrm{s}), 1.12-1.18(4 \mathrm{H}, \mathrm{m}), 1.41-1.51(2 \mathrm{H}, \mathrm{m}), 1.57-1.61$ $(2 \mathrm{H}, \mathrm{m}), 1.76(3 \mathrm{H}, \mathrm{s}), 2.01-2.07(1 \mathrm{H}, \mathrm{m}), 2.13-2.25(2 \mathrm{H}, \mathrm{m}), 2.43(1 \mathrm{H}, \mathrm{dd}, J=4.0,16.5 \mathrm{~Hz})$, 2.48-2.59 (2H, m), $2.82(1 \mathrm{H}, \mathrm{dd}, J=9.5,13.5 \mathrm{~Hz}), 3.36(3 \mathrm{H}, \mathrm{s}), 3.37(3 \mathrm{H}, \mathrm{s}), 3.38-3.42(2 \mathrm{H}, \mathrm{m})$, $3.43(3 \mathrm{H}, \mathrm{s}), 3.44(3 \mathrm{H}, \mathrm{s}), 3.56-3.62(1 \mathrm{H}, \mathrm{m}), 3.64(1 \mathrm{H}, \mathrm{dd}, J=5.0,9.0 \mathrm{~Hz}), 3.85-3.89(1 \mathrm{H}, \mathrm{m})$, $3.95(1 \mathrm{H}, \mathrm{dd}, J=2.5,8.5 \mathrm{~Hz}), 4.21(1 \mathrm{H}, \mathrm{dd}, J=2.5,9.0 \mathrm{~Hz}), 4.26(1 \mathrm{H}, \mathrm{t}, J=9.0 \mathrm{~Hz}), 4.54-4.61$ $(2 \mathrm{H}, \mathrm{m}), 4.64(1 \mathrm{H}, \mathrm{d}, J=6.5 \mathrm{~Hz}), 4.67(1 \mathrm{H}, \mathrm{d}, J=6.5 \mathrm{~Hz}), 4.70-4.75(2 \mathrm{H}, \mathrm{m}), 4.83(1 \mathrm{H}, \mathrm{d}, J=$ $7.0 \mathrm{~Hz}), 4.97(1 \mathrm{H}, \mathrm{d}, J=9.5 \mathrm{~Hz}), 5.50(1 \mathrm{H}, \mathrm{s}), 5.65(1 \mathrm{H}, \mathrm{d}, J=4.5 \mathrm{~Hz}), 7.27-7.39(5 \mathrm{H}, \mathrm{m}) ;{ }^{13} \mathrm{C}$ NMR $\left(125 \mathrm{MHz}, \mathrm{CDCl}_{3}\right) \delta_{\mathrm{C}} 12.17,12.21,18.22,18.53,21.38,22.00,24.80,35.36,37.31,37.69$, $38.12,41.21,42.33,45.36,55.95,56.00,56.26,56.71,58.89,66.46,66.72,67.32,75.44,75.72$, $78.00,81.52,97.84,98.97,103.57,127.69,129.29,129.74,131.06,135.39,139.61,153.51$, $170.76,182.62,193.62$; 
Compound 11:
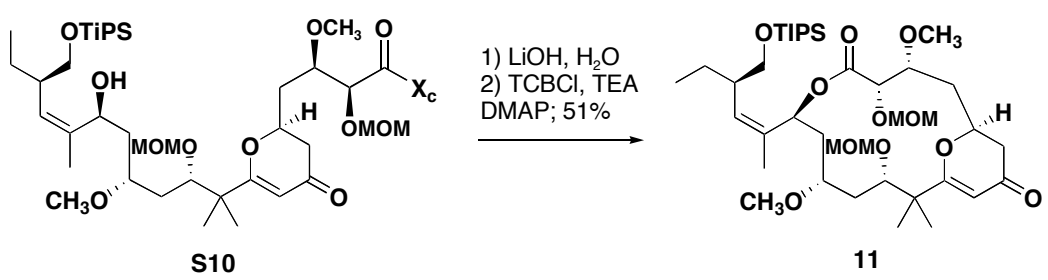

To a stirred solution of $\mathbf{S 1 0}$ (42mg, $0.045 \mathrm{mmol}$ ) in $2 \mathrm{ml}$ THF was added $0.3 \mathrm{ml} \mathrm{H}_{2} \mathrm{O}$ and aq. $\mathrm{LiOH}\left(1 \mathrm{M}\right.$ in $\left.\mathrm{H}_{2} \mathrm{O}, 0.09 \mathrm{ml}, 0.09 \mathrm{mmol}\right) 0^{\circ} \mathrm{C}$, the mixture was stirred at the same temperature for 40 minutes. After TLC shows the completion of reaction, the solution was acidified with acetic acid (adjust to $\mathrm{PH}=3$ ) and extracted with ethyl acetate. The organic layers were combined, dried $\left(\mathrm{Na}_{2} \mathrm{SO}_{4}\right)$ and evaporated to give the corresponding seco-acid, which was then dissolved in $1 \mathrm{ml}$ THF. To this solution was added $\mathrm{Et}_{3} \mathrm{~N}(70 \mathrm{mg}, 0.69 \mathrm{mmol})$ and trichlorobenzoyl chloride $(66 \mathrm{mg}$, $0.27 \mathrm{mmol})$ at $0^{\circ} \mathrm{C}$. The reaction mixture was warmed up to room temperature and stirred at the same temperature for 2 hours. Toluene $(15 \mathrm{ml})$ was added to the reaction mixture and the resulting diluted solution was added (via syringe pump, $3 \mathrm{ml} / \mathrm{h}$ ) to a solution of DMAP $(114 \mathrm{mg}, 0.93 \mathrm{mmol})$ in toluene $(20 \mathrm{ml})$ at room temperature. After continued stirring for 12 hours, the reaction mixture was heated to $90^{\circ} \mathrm{C}$ and stirred at the same temperature for 6 hour. Aqueous $\mathrm{NaHCO}_{3}$ was added and extracted with ethyl acetate, the organic layers were combined, dried $\left(\mathrm{Na}_{2} \mathrm{SO}_{4}\right)$ and evaporated to give a residue, which was subjected to column chromatography to afford (-)-11 $\left(18 \mathrm{mg}, 56 \%, 2\right.$ steps) as a colorless oil, $[\alpha]_{\mathrm{D}}^{22}=-81.2^{\circ}\left(\mathrm{c} 0.005, \mathrm{CHCl}_{3}\right)$; IR (thin film, $\left.\mathrm{cm}^{-1}\right) 680$, $805,882,922,1033,1096,1153,1243,1332,1391,1463,1595,1667,1731,1759,2866,2941$; ${ }^{1} \mathrm{H}$ NMR $\left(500 \mathrm{MHz}, \mathrm{CDCl}_{3}\right) \delta_{\mathrm{H}} 0.88(3 \mathrm{H}, \mathrm{t}, J=7.5 \mathrm{~Hz}), 1.02-1.11(21 \mathrm{H}, \mathrm{m}), 1.19(3 \mathrm{H}, \mathrm{s}), 1.20$ $(3 \mathrm{H}, \mathrm{s}), 1.23-1.30(1 \mathrm{H}, \mathrm{m}), 1.45-1.62(2 \mathrm{H}, \mathrm{m}), 1.71(3 \mathrm{H}, \mathrm{s}), 1.66-1.82(2 \mathrm{H}, \mathrm{m}), 1.95-2.05(2 \mathrm{H}$, $\mathrm{m}), 2.23(1 \mathrm{H}, \mathrm{ddd}, J=4.0,7.0,15.0 \mathrm{~Hz}), 2.37(1 \mathrm{H}, \mathrm{dd}, J=2.5,16.5 \mathrm{~Hz}), 2.56(1 \mathrm{H}, \mathrm{dd}, J=14.0$, $16.5 \mathrm{~Hz}), 2.58-2.64(1 \mathrm{H}, \mathrm{m}), 3.30(3 \mathrm{H}, \mathrm{s}), 3.40(3 \mathrm{H}, \mathrm{s}), 3.40-3.46(1 \mathrm{H}, \mathrm{m}), 3.41(3 \mathrm{H}, \mathrm{s}), 3.48(3 \mathrm{H}$, s), 3.51-3.62 (2H, m), 3.84-3.91 (2H, m), $4.18(1 \mathrm{H}, \mathrm{d}, J=7.0 \mathrm{~Hz}), 4.28-4.35(1 \mathrm{H}, \mathrm{m}), 4.67-4.75$ $(4 \mathrm{H}, \mathrm{m}), 5.20(1 \mathrm{H}, \mathrm{d}, J=10.0 \mathrm{~Hz}), 5.53(1 \mathrm{H}, \mathrm{s}), 5.80(1 \mathrm{H}, \mathrm{dd}, J=4.5,6.5 \mathrm{~Hz}) ;{ }^{13} \mathrm{C}$ NMR $(75$ $\left.\mathrm{MHz}, \mathrm{CDCl}_{3}\right) \delta_{\mathrm{C}} 12.21,18.18,18.29,20.53,24.70,25.15,29.96,34.84,36.34,38.45,40.99$, $41.97,45.15,56.22,56.71,58.33,65.89,70.63,74.67,76.42,76.75,77.47,79.16,85.01,96.35$, 99.37, 105.17, 131.54, 133.11, 169.39, 180.99, 193.35; HRMS calc. for $\mathrm{C}_{39} \mathrm{H}_{70} \mathrm{NO}_{11} \mathrm{Si}[\mathrm{M}+\mathrm{H}]^{+}$ 743.4766, found 743.4744 .

Peloruside A:
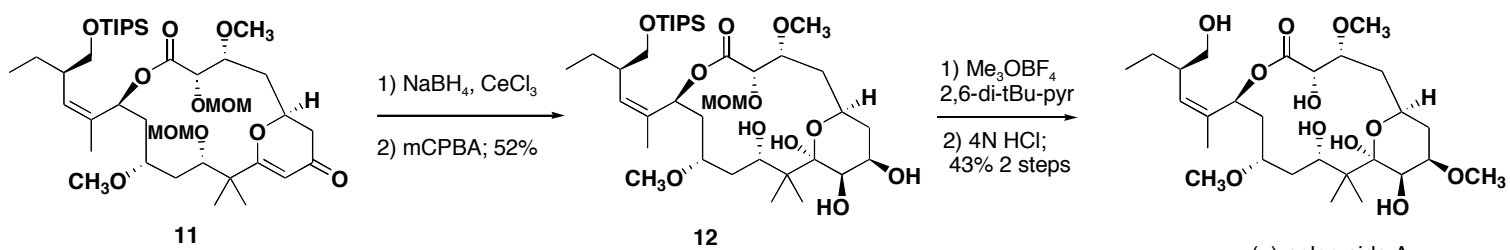

(+)-peloruside A

To a stirred solution of $\mathbf{1 1}(15 \mathrm{mg}, 0.020 \mathrm{mmol})$ and $\mathrm{CeCl}_{3} \cdot 7 \mathrm{H}_{2} \mathrm{O}(7.5 \mathrm{mg}, 0.020 \mathrm{mmol})$ in $\mathrm{MeOH}(2 \mathrm{ml})$ was added $\mathrm{NaBH}_{4}(0.95 \mathrm{mg}, 0.025 \mathrm{mmol})$ at $-60^{\circ} \mathrm{C}$, the reaction mixture was stirred at the same temperature for 40 minutes and quenched with brine. The mixture was warmed up to room temperature, extracted with ethyl acetate, the organic layers were combined, dried $\left(\mathrm{Na}_{2} \mathrm{SO}_{4}\right)$ and evaporated to give the crude alcohol which was used for the next step immediately without further purification due to instability.

The crude alcohol prepared above was dissolved in $3 \mathrm{ml} \mathrm{CH}_{2} \mathrm{Cl}_{2}$ and treated with solid 
$\mathrm{NaHCO}_{3}(9 \mathrm{mg}, 0.11 \mathrm{mmol})$. To this stirred mixture, purified mCPBA $(3.6 \mathrm{mg}, 0.021 \mathrm{mmol})$ was added at $-30^{\circ} \mathrm{C}$. The mixture was stirred at the same temperature for 30 minutes and quenched with aqueous $\mathrm{NH}_{4} \mathrm{Cl}$. Extracted with ethyl acetate, the organic layers were combined, dried $\left(\mathrm{Na}_{2} \mathrm{SO}_{4}\right)$ and evaporated to give a residue which was subjected to a column chromatography to afford alcohol (-)-12 (7.5mg, 52\% for 2 steps) as a colorless oil, $[\alpha]_{\mathrm{D}}^{22}=-66.7^{\circ}\left(\mathrm{c} 0.004, \mathrm{CHCl}_{3}\right)$; IR (thin film, $\mathrm{cm}^{-1}$ ) 680, 805, 882, 920, 1034, 1097, 1152, 1210, 1382, 1463, 1753, 2866, 2942, 3391; ${ }^{1} \mathrm{H}$ NMR $\left(600 \mathrm{MHz}, \mathrm{CD}_{3} \mathrm{OD}\right) \delta_{\mathrm{H}} 0.88(3 \mathrm{H}, \mathrm{t}, J=7.8 \mathrm{~Hz}), 1.02-1.08(21 \mathrm{H}, \mathrm{m}), 1.12(6 \mathrm{H}, \mathrm{s})$, 1.18-1.23 $(1 \mathrm{H}, \mathrm{m}), 1.25-1.32(1 \mathrm{H}, \mathrm{m}), 1.40-1.50(1 \mathrm{H}, \mathrm{m}), 1.53(1 \mathrm{H}, \mathrm{ddd}, J=2.4,4.2,12.0 \mathrm{~Hz})$, $1.67(3 \mathrm{H}, \mathrm{s}), 1.63-1.73(1 \mathrm{H}, \mathrm{m}), 1.85(1 \mathrm{H}, \mathrm{dd}, J=12.6,15.0 \mathrm{~Hz}), 1.81-1.92(1 \mathrm{H}, \mathrm{m}), 1.94-2.08$ $(1 \mathrm{H}, \mathrm{m}), 2.14(1 \mathrm{H}, \mathrm{ddd}, J=2.4,6.6,15.0 \mathrm{~Hz}), 2.26-2.36(1 \mathrm{H}, \mathrm{m}), 2.46-2.54(1 \mathrm{H}, \mathrm{m}), 3.38(3 \mathrm{H}, \mathrm{s})$, $3.39(6 \mathrm{H}, \mathrm{s}), 3.34-3.46(3 \mathrm{H}, \mathrm{m}), 3.57-3.62(1 \mathrm{H}, \mathrm{m}), 3.73(1 \mathrm{H}, \mathrm{d}, J=3.0 \mathrm{~Hz}), 3.84-3.92(1 \mathrm{H}, \mathrm{m})$, 3.94-4.01 (2H, m), $4.08(1 \mathrm{H}, \mathrm{ddd}, J=3.0,4.8,11.4 \mathrm{~Hz}), 4.26-4.42(1 \mathrm{H}, \mathrm{m}), 4.51-4.62(1 \mathrm{H}, \mathrm{m})$, 4.63-4.71 (3H, m), $5.01(1 \mathrm{H}, \mathrm{d}, J=10.2 \mathrm{~Hz}), 5.47(1 \mathrm{H}, \mathrm{br} \mathrm{s})$.

To a stirred solution of $12(5 \mathrm{mg}, 0.0068 \mathrm{mmol})$ in $\mathrm{CH}_{2} \mathrm{Cl}_{2}(2.5 \mathrm{ml})$ was added 2.6-di-tert-butyl pyridine $(28 \mathrm{mg}, 0.15 \mathrm{mmol})$ and $\mathrm{Me}_{3} \mathrm{OBF}_{4}(15 \mathrm{mg}, 0.1 \mathrm{mmol})$ at $0^{\circ} \mathrm{C}$. The resulting mixture was stirred at $0^{\circ} \mathrm{C}$ until TLC shows the completion of reaction. Aqueous $\mathrm{NaHCO}_{3}$ was added, extracted with ethyl acetate. The organic layers were combined, dried $\left(\mathrm{Na}_{2} \mathrm{SO}_{4}\right)$ and evaporated to give a residue, which was filtered through a short column (silica gel) to provide a crude material. This material was then dissolved in THF $(1.5 \mathrm{ml})$ and treated with $4 \mathrm{~N} \mathrm{HCl}(1.5 \mathrm{ml})$ at $0^{\circ} \mathrm{C}$, the resulting mixture was warmed up to room temperature and stirred at the same temperature for 3 hours. The reaction was quenched by aqueous $\mathrm{NaHCO}_{3}$ and extracted with ethyl acetate, the organic layers were combined, dried $\left(\mathrm{Na}_{2} \mathrm{SO}_{4}\right)$ and evaporated to give a residue which was subjected to a column chromatography to provide ( + )-peloruside $\mathrm{A}(1.6 \mathrm{mg}, 43 \%$ for 2 steps) as a colorless oil, $[\alpha]^{22}{ }_{\mathrm{D}}=+16.5^{\circ}$ (c $\left.0.0015, \mathrm{CH}_{2} \mathrm{Cl}_{2}\right)$; (lit.: ${ }^{2}[\alpha]^{20}{ }_{\mathrm{D}}=+16^{\circ}\left(\mathrm{c} 0.30, \mathrm{CH}_{2} \mathrm{Cl}_{2}\right)$ ); IR (thin film, $\left.\mathrm{cm}^{-1}\right)$ 1085, 1155, 1222, 1383, 1456, 1739, 2850, 2929, 3445; ${ }^{1} \mathrm{H}$ NMR (500 MHz, $\left.\mathrm{CDCl}_{3}\right)$ $\delta_{\mathrm{H}} 0.86(3 \mathrm{H}, \mathrm{t}, J=7.5 \mathrm{~Hz}), 1.10(3 \mathrm{H}, \mathrm{s}), 1.12(3 \mathrm{H}, \mathrm{s}), 1.16-1.21(1 \mathrm{H}, \mathrm{m}), 1.40-1.46(2 \mathrm{H}, \mathrm{m}), 1.53$ $(1 \mathrm{H}, \mathrm{q}, J=12.0 \mathrm{~Hz}), 1.68(3 \mathrm{H}, \mathrm{d}, J=1.0 \mathrm{~Hz}), 1.75-1.82(1 \mathrm{H}, \mathrm{m}), 1.79(1 \mathrm{H}, \mathrm{ddd}, J=2.5,5.0,12.5$ $\mathrm{Hz}), 2.00-2.18(4 \mathrm{H}, \mathrm{m}), 2.56-2.66(1 \mathrm{H}, \mathrm{m}), 2.71(1 \mathrm{H}, \mathrm{d}, J=9.0 \mathrm{~Hz}), 3.01(1 \mathrm{H}, \mathrm{dd}, J=3.0,8.0$ $\mathrm{Hz}), 3.31(3 \mathrm{H}, \mathrm{s}), 3.36,(1 \mathrm{H}, \mathrm{t}, J=10.5 \mathrm{~Hz}), 3.39(3 \mathrm{H}, \mathrm{s}), 3.48(3 \mathrm{H}, \mathrm{s}), 3.65(1 \mathrm{H}, \mathrm{ddd}, J=3.0$, 7.5, 10.0 Hz), $3.82(1 \mathrm{H}, \mathrm{ddd}, J=3.0,5.0,11.5 \mathrm{~Hz}), 3.96-4.02(1 \mathrm{H}, \mathrm{m}), 4.02(1 \mathrm{H}, \mathrm{s}), 4.22(1 \mathrm{H}, \mathrm{dd}$, $J=5.5,10.5 \mathrm{~Hz}), 4.26(1 \mathrm{H}, \mathrm{ddd}, J=2.5,4.5,10.5 \mathrm{~Hz}), 4.46(1 \mathrm{H}, \mathrm{s}), 4.53(1 \mathrm{H}, \mathrm{d}, J=9.5 \mathrm{~Hz})$, $4.91(1 \mathrm{H}, \mathrm{m}), 5.05(1 \mathrm{H}, \mathrm{d}, J=10.5 \mathrm{~Hz}), 5.69(1 \mathrm{H}, \mathrm{d}, J=10.5 \mathrm{~Hz}), 6.77(1 \mathrm{H}, \mathrm{br} . \mathrm{s}) ;{ }^{13} \mathrm{C}$ NMR $\left(125 \mathrm{MHz}, \mathrm{CDCl}_{3}\right) \delta_{\mathrm{C}} 12.24,15.84,17.45,20.81,24.60,31.67,32.59,33.92,35.70,43.37,43.60$, $55.70,56.09,59.11,63.49,66.85,67.00,70.30,70.91,73.89,75.90,77.93,78.28,101.91,131.18$, 136.09, 174.01; HRMS calc. for $\mathrm{C}_{27} \mathrm{H}_{48} \mathrm{NO}_{11}[\mathrm{M}+\mathrm{Na}]^{+}$571.3094, found 571.3079. 